\title{
Construction Sequence of the Koh Ker Monuments Constrained by the Chemical Composition and Magnetic Susceptibility of Its Bricks
}

\author{
Etsuo Uchida, Yuichiro Sakurai \\ Department of Resources and Environmental Engineering, Waseda University, Tokyo, Japan \\ Email: weuchida@waseda.jp
}

How to cite this paper: Uchida, E., \& Sakurai, Y. (2018). Construction Sequence of the Koh Ker Monuments Constrained by the Chemical Composition and Magnetic Susceptibility of Its Bricks. Archaeological Discovery, 6, 173-185.

https://doi.org/10.4236/ad.2018.62009

Received: March 31, 2018

Accepted: April 27, 2018

Published: April 30, 2018

Copyright ( 92018 by authors and Scientific Research Publishing Inc. This work is licensed under the Creative Commons Attribution International License (CC BY 4.0).

http://creativecommons.org/licenses/by/4.0/ (c) (i) Open Access

\begin{abstract}
Using a portable X-ray fluorescence analyzer and magnetic susceptibility meter, we measured fired bricks from nine temples (Prasat Thom, Prasat Damrei, Prasat Chamreh, Prasat Krachap, Prasat Kraham, Prasat Chen, Prasat Banteay Pir Chan, Prasat Chrap, and Prasat Pram) of the Koh Ker monuments, Cambodia. Based on cluster analysis of the chemical compositional data for Ti, Fe, $\mathrm{Rb}, \mathrm{Y}$, and $\mathrm{Zr}$, as well as magnetic susceptibility data, the brick buildings could be classified into four groups (Stages A to D). Taking into consideration the five construction stages (Stages (1) to (5)) defined by Uchida et al. (2014) for laterite buildings, and using the assumption that all buildings were basically constructed outwards from the center, we define a construction sequence for these brick buildings. Thus, the following chronological relationship was obtained for construction stages of both laterite and brick buildings: Stage $\mathrm{A} \rightarrow$ Stages B \& (1) $\rightarrow$ Stages C \& (2) $\rightarrow$ Stages D \& (3) $\rightarrow$ Stage (4) $\rightarrow$ Stage (5). We surmise that the Northern Libraries of Prasat Pram, Prasat Chen and Prasat Damrei were added after the construction of their Inner Enclosures.
\end{abstract}

\section{Keywords}

Koh Ker Monuments, Bricks, Portable X-Ray Fluorescence Analyzer, Magnetic Susceptibility, Construction Sequence, Cambodia

\section{Introduction}

The Angkor monuments are representative of the monuments constructed by the Khmer people from the 9th to 15th century. The Angkor area was the capital of the Khmer Empire, situated north of Tonle Sap Lake. The Koh Ker monu- 
ments are located $85 \mathrm{~km}$ northeast of the Angkor monuments (Figure 1). Koh Ker was the temporary capital of the Khmer Empire, governed by Jayavarman IV from 921 or 928 to 941 AD (Jacques \& Lafond, 2004). To date, 76 temples have been confirmed to form the Koh Ker monuments (Mizoguchi \& Nakagawa, 2011). The Koh Ker monuments are situated along the northeast Royal Road, connecting the Angkor monuments to the Wat Phu temple complex in Southern Laos. The northeast Royal Road passes 6 km northwest of the Koh Ker monuments. Prasat Thom is the largest temple of the Koh Ker monuments. A stepped pyramid called Prang is situated at the westernmost end of Prasat Thom. The main axis of the temples of the Koh Ker monuments is rotated, around $0^{\circ}$ to $22^{\circ}$ from an east-west axis, in an anticlockwise direction.

Investigation of the Koh Ker monuments began with Harmand (1879), and was followed by Delaporte (1880), Aymonier (1900), Lunet de Lajonquière (1902), Groslier (1924-1926), Parmentier (1939), Jacques \& Lafond (2004), Evans (2010-2011), and Mizoguchi \& Nakagawa (2011). To date, no study except for Uchida et al. (2014) has focused on the construction sequence of the Koh Ker monuments.

Sandstone, laterite, and bricks were the major construction materials used in the Koh Ker monuments. Sandstone blocks were used to the Inner Enclosure, East and West Gopuras of the Middle Enclosure, and East Gopura of Prasat Thom, Prasat Balang, Prasat Thneng, Prasat G, Prasat Khna and other buildings. Sandstone blocks were supplied from the surrounding area (Evans, 2010-2011). Because little difference in the chemical composition or magnetic susceptibility of these sandstone blocks was found, sandstone could not be used to determine the construction sequence of the Koh Ker monuments (Uchida et al., 2014). In contrast, there are remarkable differences in the chemical composition and magnetic susceptibility of the laterite used in construction of many of its buildings.

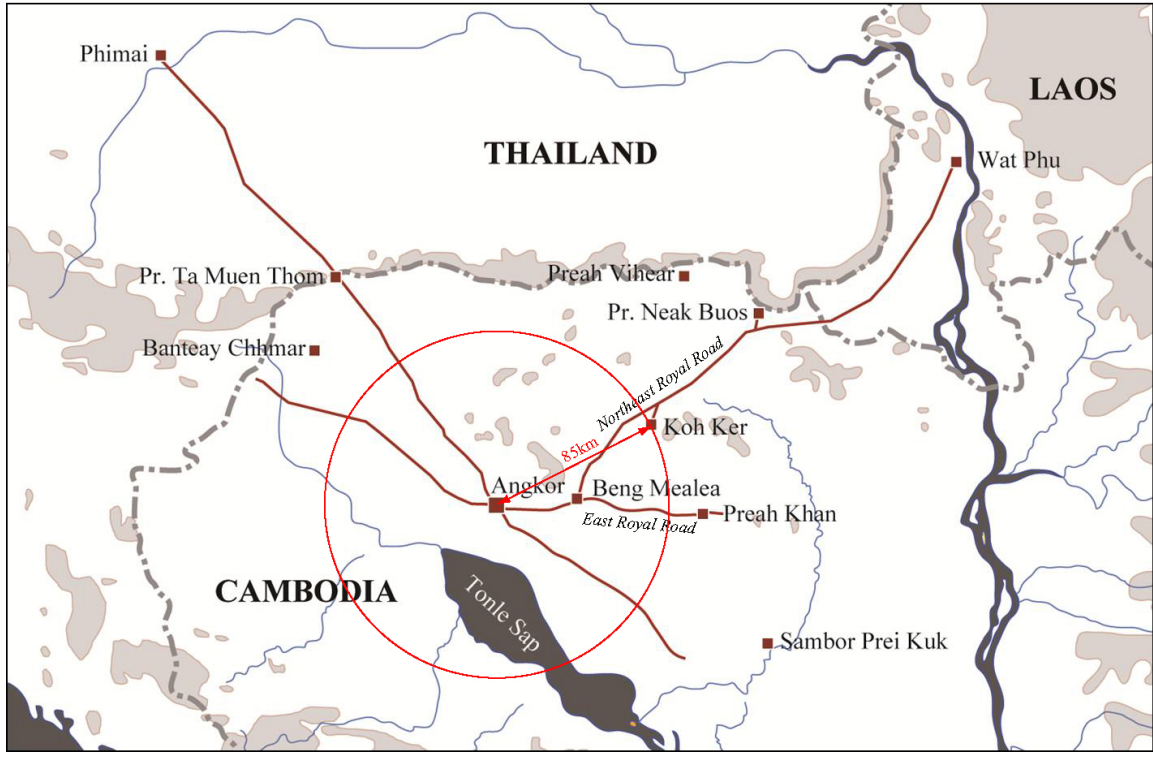

Figure 1. Map showing the location of the Koh Ker monuments in Cambodia. 
Uchida et al. (2014) determined the construction sequence of the laterite buildings in the Koh Ker monuments based on Sr content and magnetic susceptibility of these laterite components. As a result, five construction stages were recognized among the laterite buildings. In this study, we focus on the bricks, another important construction material of the Koh Ker monuments, to deduce the construction sequence of the brick buildings. All bricks used in the Khmer monuments, including the Koh Ker monuments, are fired bricks. Bricks were used for the Sanctuaries of Prasat Thom, Prasat Damrei, Prasat Chamreh and Prasat Krachap, and the Libraries of many temples (Figure 2 \& Figure 3). The sizes of these bricks range from 250 to $320 \mathrm{~mm}$ in length, 130 to $190 \mathrm{~mm}$ in width, and 52 to $71 \mathrm{~mm}$ in thickness.

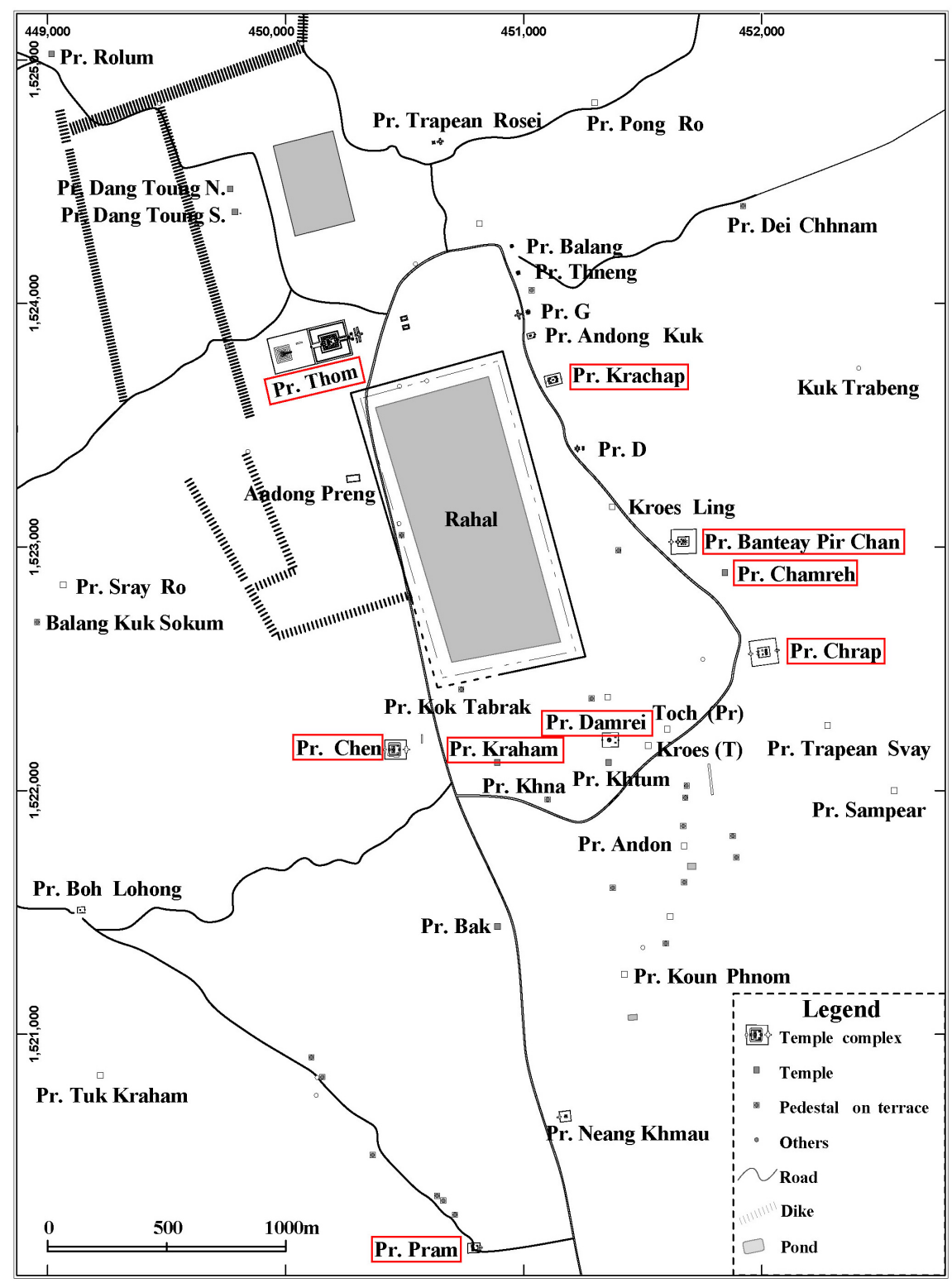

Figure 2. Distribution of major temples of the Koh Ker monuments at the study site. 

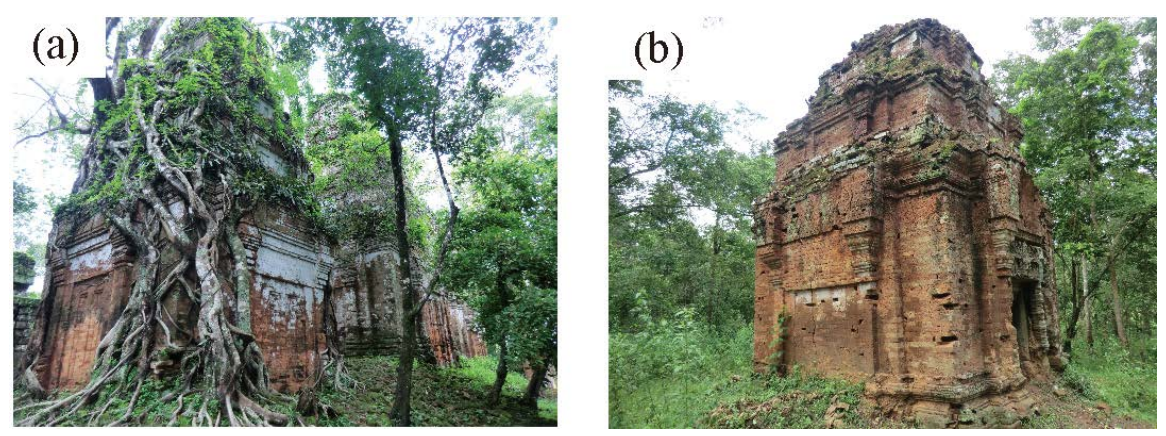

Figure 3. Brick buildings of the Koh Ker monuments. (a) Sanctuaries of Prasat Pram; and (b) Central Sanctuary of Prasat Chamreh.

\section{Methods}

In this study, we conducted chemical analyses and magnetic susceptibility measurements of bricks used in the Koh Ker monuments. Measurements were carried out on brick buildings from the following nine temples: Prasat Thom, Prasat Damrei, Prasat Chamreh, Prasat Krachap, Prasat Kraham, Prasat Chen, Prasat Banteay Pir Chan, Prasat Chrap, and Prasat Pram (Figure 2 \& Figure 3).

Analyses of the bricks were conducted non-destructively, using a portable X-ray fluorescence (XRF) analyzer (Delta Premium; Innov-X Systems Inc., Waltham, MA, USA). "Soil mode" was used for all measurements. Prior to analysis, calibration curves were obtained using Japanese standard rock samples: JA-1, JA-2, JB-1b, JB-2, JB-3, JG-1a, JG-2, JGb-1, JR-1, and JR-2 (Imai et al., 1995). The total measurement time was fixed at $60 \mathrm{~s}$. Field measurements were conducted on the surfaces of 10 bricks, not covered with soil, lichen or algae, from each building, and an average value was calculated.

Given that magnetic susceptibility of the sandstones used in the Angkor monuments was very useful in determining the construction sequence of the sandstone buildings and the provenance of the sandstone blocks (Uchida et al., 2003, 2007, 2013), we applied this technique to bricks in this study. The magnetic susceptibility measurements were conducted non-destructively, using a portable magnetic susceptibility meter (SM30; ZH Instruments, Brno, Czech Republic). We measured brick surfaces of $5 \mathrm{~cm} \times 5 \mathrm{~cm}$. Contribution to measured magnetic susceptibility is around $50 \%$ at a depth of $5 \mathrm{~mm}$ from the surface, around $80 \%$ at a depth of $15 \mathrm{~mm}$, and around $90 \%$ at a depth of $25 \mathrm{~mm}$. The measurement time was about $2 \mathrm{~s}$, giving an accuracy of $1 \times 10^{-6}$ SI unit. Magnetic susceptibility measurements were made on 50 bricks at each building, and an average value was calculated. Measurements were carried out on flat surfaces of bricks, not covered with soil, lichen or algae.

The bricks in the Koh Ker monuments have a yellowish to reddish brown color on surface. This color may reflect the existence of hematite and/or goethite. The bricks in the Koh Ker monuments frequently contain a small amount of pisolites, up to $10 \mathrm{~mm}$ in diameter, in which iron and aluminum oxides are concentrated. 
In addition to the chemical analyses and magnetic susceptibility measurements of the bricks, a cluster analysis (Ward's method) was carried out using these data to obtain groupings of the brick buildings in the Koh Ker monuments.

\section{Results}

\subsection{General Chemical Composition}

Our XRF measurements detected the following common elements in bricks of the Koh Ker monuments: Ti, Fe, Rb, Y, Zr, Sr, Mn, Ca, V, Co, Cu, Zn, and Pb. Average compositions of selected elements are summarized in Table 1.

\subsection{General Magnetic Susceptibility}

Average magnetic susceptibilities of bricks of the Koh Ker monuments are summarized in Table 1. Bricks used in the East Gopura of Prasat Chen had the lowest average magnetic susceptibility $\left(0.58 \times 10^{-3} \mathrm{SI}\right.$ units), while those in Prasat Kraham had the highest value $\left(4.99 \times 10^{-3} \mathrm{SI}\right.$ units). The average value for bricks used in the Koh Ker monuments was $2.61 \times 10^{-3} \mathrm{SI}$ units. There was no correlation found between magnetic susceptibility and the Fe content (correlation coefficient: -0.08 ).

\subsection{Cluster Analysis}

The 13 elements most commonly detected in bricks of the Koh Ker monuments were: $\mathrm{Ti}, \mathrm{Fe}, \mathrm{Rb}, \mathrm{Y}, \mathrm{Zr}, \mathrm{Sr}, \mathrm{Mn}, \mathrm{Ca}, \mathrm{V}, \mathrm{Co}, \mathrm{Cu}, \mathrm{Zn}$, and $\mathrm{Pb}$. There were no significant differences in the contents of $\mathrm{Co}, \mathrm{Cu}$ and $\mathrm{Zn}$ among buildings. However, $\mathrm{Ca}, \mathrm{Sr}, \mathrm{Mn}, \mathrm{Pb}$, and $\mathrm{V}$ showed large fluctuations within the same buildings. Therefore, our cluster analysis using Ward's method, was restricted to the elements, Ti, Fe, Rb, Y, and Zr, as well as magnetic susceptibility (Table 1).

The dendrogram obtained using chemical compositional data for $\mathrm{Ti}, \mathrm{Fe}, \mathrm{Rb}$, $\mathrm{Y}$, and $\mathrm{Zr}$, as well as magnetic susceptibility data is shown in Figure 4. Using a distance of around two in the dendrogram (Figure 4), the brick buildings in the Koh Ker monuments could be classified into four groups, demarcated as Stages A to D, respectively. All the buildings in Stage A belong to Prasat Thom. The bricks used in Stage A are characterized by a high Y content (more than $33 \mathrm{ppm}$ ) (Table 1). Prasat Damrei, Prasat Chen, Prasat Chamreh and Prasat Kraham of Prasat Thom belong to Stage B. The bricks of Stage B are rich in $\mathrm{Rb}$ (more than 39 ppm). Stage $C$ includes Prasat Chen and Prasat Kraham. The bricks of Stage $\mathrm{C}$ are considerably richer in $\mathrm{Zr}$ (more than $540 \mathrm{ppm}$ ), compared with other stages. Stage D consists of Prasat Banteay Pir Chan and Prasat Pram. In addition, the Northern Libraries of Prasat Chen, Prasat Chrap and Prasat Damrei belong to Stage D. The bricks of Stage $\mathrm{D}$ are depleted in Rb (less than $36 \mathrm{ppm}$ ). No systematic difference was observed in the magnetic susceptibility of the bricks among the various buildings. Based on our cluster analysis, we outline a construction sequence for the brick buildings below. 
Table 1. Average $\mathrm{Ti}, \mathrm{Fe}, \mathrm{Rb}, \mathrm{Y}$, and $\mathrm{Zr}$ contents determined using a portable $\mathrm{X}$-ray fluorescence analyzer and average magnetic susceptibilities of bricks in the Koh Ker monuments. s.d.: standard deviation (1 $\sigma$ ), and M.S.: magnetic susceptibility.

\begin{tabular}{|c|c|c|c|c|c|c|c|c|c|}
\hline Stage & Temple & Building & & $\begin{array}{c}\mathrm{Ti} \\
\mathrm{ppm}\end{array}$ & $\begin{array}{c}\mathrm{Fe} \\
\mathrm{ppm}\end{array}$ & $\begin{array}{c}\mathrm{Rb} \\
\mathrm{ppm}\end{array}$ & $\begin{array}{c}\mathrm{Y} \\
\mathrm{ppm}\end{array}$ & $\begin{array}{c}\mathrm{Zr} \\
\mathrm{ppm}\end{array}$ & $\begin{array}{l}\text { M.S. } \\
10^{-3} \mathrm{SL}\end{array}$ \\
\hline \multirow{8}{*}{ A } & \multirow{2}{*}{ Pr. Thom } & \multirow{2}{*}{ N Library } & average & 2783 & 24205 & 40 & 38 & 250 & 1.81 \\
\hline & & & s.d. $(1 \sigma)$ & (509) & $(3225)$ & $(5)^{1}$ & $(2)^{\top}$ & $(31)^{\top}$ & $(1.95)$ \\
\hline & \multirow{2}{*}{ Pr. Thom } & \multirow{2}{*}{$\begin{array}{l}\text { Sanctuary situated in the } \\
\text { NE of Central Sanctuary }\end{array}$} & average & 2561 & 19827 & 44 & 34 & 190 & 2.85 \\
\hline & & & s.d. $(1 \sigma)$ & (249) & $(4491)^{1}$ & $(13)^{T}$ & $(6)^{1}$ & $(21)^{\prime}$ & $(2.41)$ \\
\hline & \multirow{2}{*}{ Pr. Thom } & \multirow{2}{*}{$\begin{array}{c}\text { Central Sanctuary } \\
\text { (Mandapa) }\end{array}$} & average & 2514 & 21530 & 29 & 33 & 271 & 1.53 \\
\hline & & & s.d. $(1 \sigma)$ & $(439)$ & $(7293)$ & (3) & $(10)^{\prime}$ & $(122)$ & $(1.90)$ \\
\hline & \multirow{2}{*}{ Pr. Thom } & \multirow{2}{*}{ Central Sanctuary (Main) } & average & 2178 & 19998 & 30 & 40 & 207 & 4.06 \\
\hline & & & s.d. $(1 \sigma)$ & $(341)$ & $(6029)^{\prime}$ & $(4)^{\prime}$ & $(14)^{\prime}$ & $(25)^{\prime}$ & $(2.58)$ \\
\hline \multirow{18}{*}{ B } & \multirow{2}{*}{ Pr. Damrei } & \multirow{2}{*}{ S Library } & average & 2793 & 22410 & 48 & 13 & 239 & 1.60 \\
\hline & & & s.d. $(1 \sigma)$ & $(423)$ & $(2502)$ & $(4)^{\prime}$ & $(3)^{\prime}$ & $(36)^{\prime}$ & $(1.67)$ \\
\hline & \multirow{2}{*}{ Pr. Chamreh } & \multirow{2}{*}{ Sanctuary } & average & 2731 & 28067 & 46 & 22 & 184 & 1.47 \\
\hline & & & s.d. $(1 \sigma)$ & (312) & $(5851)^{\prime}$ & $(10)^{\top}$ & $(6)^{\top}$ & $(49)^{7}$ & $(2.33)$ \\
\hline & \multirow{2}{*}{ Pr. Damrei } & F Gopura & average & 3423 & 29564 & 45 & 17 & 187 & 2.45 \\
\hline & & E Gopura & s.d. $(1 \sigma)$ & (473) & $(1995)$ & $(8)^{\prime}$ & (4) & $(29)^{\prime}$ & $(2.10)$ \\
\hline & Pr Damrei & W Gonura & average & 2986 & 22821 & 48 & 11 & 235 & 3.03 \\
\hline & M. Daाmet & Wuptila & s.d. $(1 \sigma)$ & (299) & $(4565)$ & $(10)$ & (2) & $(35)^{\prime}$ & $(2.96)$ \\
\hline & Pr. Damrei & Central Sanctuary & average & 3213 & 25385 & 67 & 16 & 282 & 2.45 \\
\hline & F1. Danter & Central sanctuary & s.d. $(1 \sigma)$ & (481) & $(4606)$ & $(8)$ & (3) & $(78)$ & $(2.79)$ \\
\hline & Pr. Chen & W Gopura & average & 3802 & 30141 & 60 & 22 & 304 & 3.38 \\
\hline & P1. Cient & & s.d. $(1 \sigma)$ & (346) & $(4302)$ & $(7)^{\prime}$ & $(7)^{\prime}$ & (69) & $(4.59)$ \\
\hline & $\operatorname{Pr}$ Chen & S Library & average & 2151 & 18686 & 39 & 18 & 476 & 1.74 \\
\hline & Pr. Cnen & S Liorary & s.d. $(1 \sigma)$ & (121) & $(1499)$ & $(7)^{\prime}$ & $(5)^{\prime}$ & $(84)^{\prime}$ & $(2.55)$ \\
\hline & Pr Thom & Pr. Kraham & average & 2913 & 24758 & 52 & 28 & 353 & 1.81 \\
\hline & 1. 1. & 11. Niantan & s.d. $(1 \sigma)$ & (639) & $(6044)$ & (14) & (5) & (140) & $(1.98)$ \\
\hline & $\operatorname{Pr}$ Chen & E Gopura & average & 2877 & 23026 & 71 & 34 & 172 & 0.58 \\
\hline & Pr. Cnen & E Gopura & s.d. $(1 \sigma)$ & (328) & (2674) & (8) & $(8)$ & (23) & $(0.78)$ \\
\hline & Pr Krachan & Central Sanctuary & average & 2860 & 19001 & 35 & 25 & 738 & 1.94 \\
\hline & 1. Nolachap & Central Sanc tuary & s.d. $(1 \sigma)$ & (365) & $(2301)$ & (6) & (5) & (278) & $(1.31)$ \\
\hline & Pr Krachan & NW I ibrary & average & 2495 & 17574 & 37 & 23 & 740 & 2.20 \\
\hline & Pr. Nactap & IN W Lioraly & s.d. $(1 \sigma)$ & (415) & $(2503)$ & (2) & (3) & (196) & $(2.21)$ \\
\hline $\mathrm{C}$ & Pr. Krachap & SW Library & average & 3061 & 21678 & 36 & 29 & 617 & 4.31 \\
\hline C & 11. Niatiap & Sw Livialy & s.d. $(1 \sigma)$ & (182) & $(3965)$ & (12) & $(9)$ & (242) & (4.14) \\
\hline & Pr. Krachan & NE Library & average & 3113 & 23409 & 45 & 29 & 545 & 3.60 \\
\hline & & & s.d. $(1 \sigma)$ & (448) & $(4393)$ & (7) & (10) & (226) & $(3.40)$ \\
\hline & Pr K nohom & Sanc & average & 2886 & 21707 & 30 & 16 & 566 & 4.99 \\
\hline & Pr. Kranam & sanciuary & s.d. $(1 \sigma)$ & (229) & (1347) & (3) & (3) & $(85)$ & $(3.46)$ \\
\hline & Pr Chran & N L ibrary & average & 2458 & 21753 & 25 & 15 & 378 & 3.86 \\
\hline & 11. сли ар & 10 Liolaly & s.d. $(1 \sigma)$ & (321) & $(2428)$ & (5) & (2) & (134) & $(3.30)$ \\
\hline & Pr. Banteay Pir & $\mathrm{N}$ tower on $\mathrm{E}$ side of & average & 2298 & 19066 & 28 & 16 & 275 & 4.71 \\
\hline & Chan & Central Sanctuary & s.d. $(1 \sigma)$ & (321) & $(3480)$ & (7) & (4) & (41) & $(4.06)$ \\
\hline & Pr. Damrei & N Library & average & 2706 & 18906 & 31 & 16 & 334 & 2.31 \\
\hline & & 14 Divialy & s.d. $(1 \sigma)$ & $(440)$ & $(3683)$ & (6) & (3) & (23) & $(1.62)$ \\
\hline & Pr. Chen & N Library & average & 2148 & 19262 & 30 & 12 & 233 & 2.75 \\
\hline & Pr. Cnen & Noliorary & s.d. $(1 \sigma)$ & (327) & $(5826)$ & $(7)$ & (2) & (47) & $(3.40)$ \\
\hline & Pr. Banteay Pir & N I ihrary & average & 2240 & 20627 & 33 & 16 & 224 & 3.22 \\
\hline & Chan & & s.d. $(1 \sigma)$ & (354) & $(2941)$ & $(6)$ & (4) & $(40)$ & $(2.67)$ \\
\hline $\mathrm{D}$ & Pr. Banteay Pir & $\mathrm{W}$ tower on $\mathrm{N}$ side of & average & 2218 & 18569 & 25 & 18 & 239 & 3.41 \\
\hline & Chan & Central Sanctuary & s.d. $(1 \sigma)$ & (306) & $(3404)$ & $(7)$ & $(18)$ & (86) & $(3.55)$ \\
\hline & Pr. Banteay Pir & S I ihrary & average & 2619 & 23840 & 36 & 23 & 178 & 3.16 \\
\hline & Chan & S Livialy & 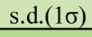 & $(92)$ & $(1745)$ & (4) & $(5)$ & $(42)$ & $(2.68)$ \\
\hline & Pr. Pram & Central Sanctuary & average & 2578 & 24169 & 27 & 21 & 158 & 2.52 \\
\hline & Pr. Fram & & s.d. $(1 \sigma)$ & (283) & $(3845)$ & (6) & (11) & $(15)$ & $(1.88)$ \\
\hline & Pr Pram & N Sanctuary & average & 2315 & 19649 & 22 & 21 & 184 & 1.51 \\
\hline & 11. 11ant & 1\% Nantuary & s.d. $(1 \sigma)$ & $(367)$ & $(3753)$ & (4) & $(8)$ & (34) & $(1.25)$ \\
\hline & Pr. Pram & S Sanctuary & average & 2197 & 21131 & 20 & 18 & 195 & 1.23 \\
\hline & & & s.d. $(1 \sigma)$ & (244) & $(1639)$ & $(6)$ & (3) & (14) & $(1.36)$ \\
\hline & Pr Pram & S I ibrary & average & 2327 & 20575 & 25 & 17 & 205 & 1.77 \\
\hline & F1. Fiam & s Livialy & s.d. $(1 \sigma)$ & (345) & $(3457)$ & (5) & $(8)$ & (48) & $(2.75)$ \\
\hline
\end{tabular}




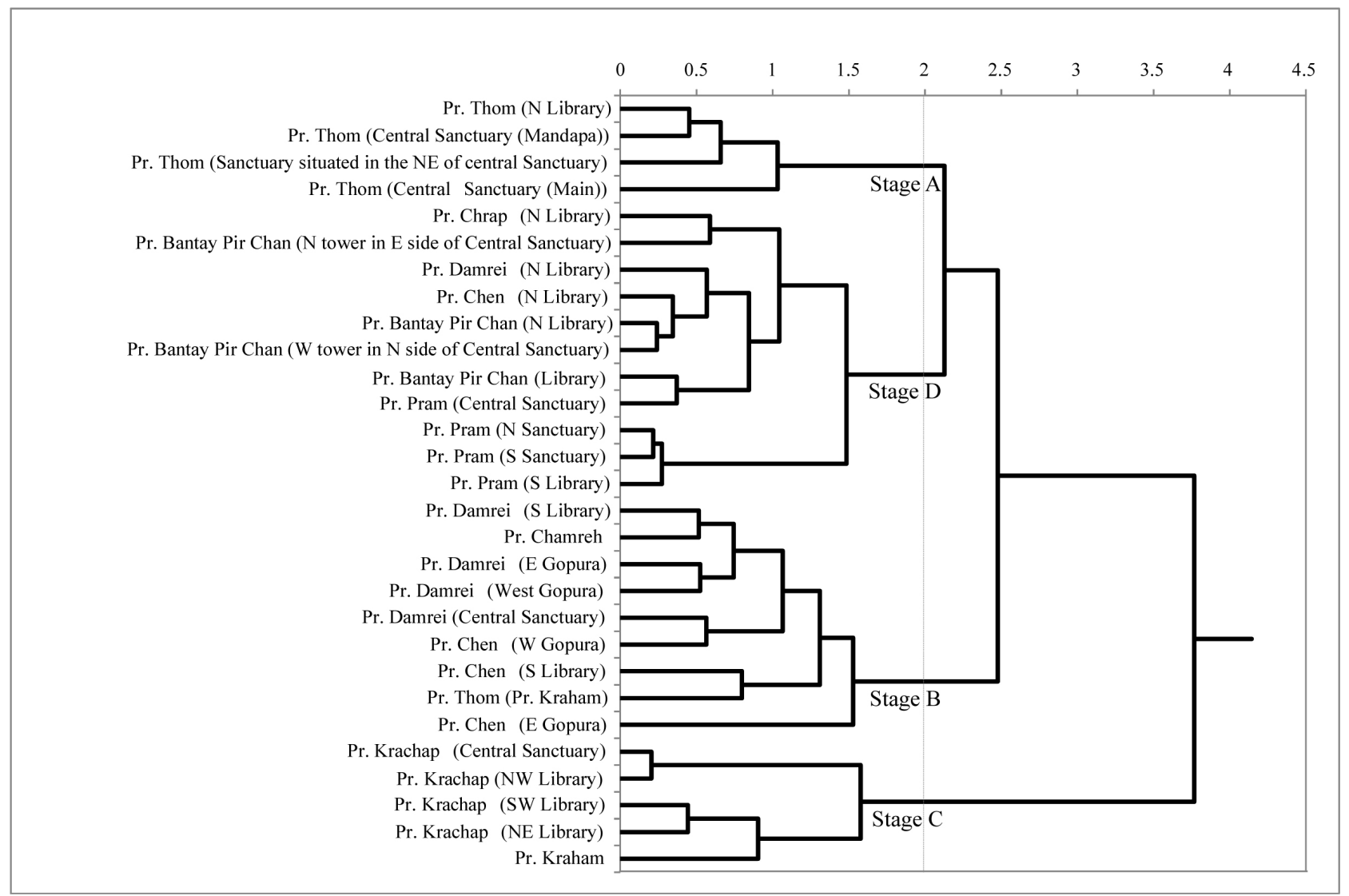

Figure 4. Dendrogram obtained from a cluster analysis (Ward's method), using chemical compositional data and magnetic susceptibility data (Table 1) of bricks from various buildings in the Koh Ker monuments. The brick buildings are classified into four groups, or Stages A to D, using a distance of 2 .

\section{Discussion of the Construction Sequence of Brick and Laterite Buildings}

Based on the chemical composition and magnetic susceptibility of laterite, Uchida et al. (2014) assigned the laterite buildings in the Koh Ker monuments to five construction stages. In particular, Uchida et al. (2014) used Sr content of the laterite to divide the laterite buildings into two groups, using a value between 300 to $400 \mathrm{ppm}$. Likewise, magnetic susceptibility of the laterite buildings was used to divide them into two groups, using a value of $2 \times 10^{-3}$ SI units. Assuming that temples were basically constructed outwards from the center, as it is demonstrated in the Angkor monuments except for middle enclosures (Dumarçay \& Groslier, 1973; Uchida et al., 2003), laterite buildings in the Koh Ker monuments were classified into five construction stages (Stages (1) to (5)) (Figure 5). Here, we discuss the relationship between the construction stages of the brick buildings (Stages A to D) obtained in this study, with those of the laterite buildings outlined in Uchida et al. (2014) (Figure 5).

All laterite buildings in Prasat Thom belong to relatively old stages (Stages (1) and (2)) of construction of the Koh Ker monuments (Figure 6). Assuming that temples were constructed outwards from the center, then the brick buildings 
(Sanctuaries and Libraries) classified as Stage A, situated inside the sandstone Inner Enclosure, were likely constructed prior to Stage (1). We surmise that the

\begin{tabular}{|c|c|c|c|c|c|c|c|c|c|c|c|c|c|c|c|c|c|c|}
\hline \multirow[b]{2}{*}{ Temple } & \multirow[b]{2}{*}{$\begin{array}{c}\text { Construction } \\
\text { materials }\end{array}$} & \multicolumn{3}{|c|}{ Sanctuary } & \multicolumn{3}{|c|}{ Middle Enclosure } & \multicolumn{3}{|c|}{ Outer Enclosure } & \multicolumn{8}{|c|}{ Other buildings } \\
\hline & & \begin{tabular}{|l|} 
Stage \\
\end{tabular} & \begin{tabular}{|l|}
$\mathrm{Sr}^{*}$ \\
$(\mathrm{l \sigma})$ \\
\end{tabular} & \begin{tabular}{|c|}
$\mathrm{MS}^{* *}$ \\
$(\mathbf{l \sigma})$
\end{tabular} & Stage & \begin{tabular}{|l|}
$\mathrm{Sr}^{*}$ \\
$(1 \sigma)$ \\
\end{tabular} & $\begin{array}{c}\mathrm{MS}^{* *} \\
(\mathrm{l \sigma})\end{array}$ & Stage & \begin{tabular}{l|}
$\mathrm{Sr}^{*}$ \\
$(1 \sigma)$
\end{tabular} & \begin{tabular}{|c|}
$\mathrm{MS}^{* *}$ \\
$(\mathrm{l \sigma})$
\end{tabular} & Stage & Buildings & $\begin{array}{l}\mathrm{Sr}^{*} \\
(\mathbf{l \sigma})\end{array}$ & \begin{tabular}{|c|}
$\mathrm{MS}^{* *}$ \\
$(\mathbf{l \sigma})$
\end{tabular} & Stage & Buildings & $\begin{array}{l}\mathrm{Sr}^{*} \\
(1 \sigma)\end{array}$ & \begin{tabular}{|c|}
$M^{* * *}$ \\
$(1 \sigma)$
\end{tabular} \\
\hline \multirow{4}{*}{ Pr. Thom } & \multirow{3}{*}{ laterite } & & & & (1) & $\begin{array}{r}29 \\
(8)\end{array}$ & \begin{tabular}{r|}
1.77 \\
$(1.36)$ \\
\end{tabular} & (1) & $\begin{array}{r}164 \\
(128) \\
\end{array}$ & $\begin{array}{r}1.69 \\
(1.22) \\
\end{array}$ & (1) & N\&S Palaces & $\begin{array}{r}94 \\
(50) \\
\end{array}$ & $\begin{array}{r}1.07 \\
(0.57) \\
\end{array}$ & (2) & Prang & $\begin{array}{r}76 \\
(18) \\
\end{array}$ & $\begin{array}{r}2.51 \\
(1.59) \\
\end{array}$ \\
\hline & & & & & & & & \multicolumn{3}{|c|}{\begin{tabular}{|c|} 
Outer Enclosure \\
surrounding Prang
\end{tabular}} & (1) & $\begin{array}{c}\begin{array}{c}\text { S Rectangular } \\
\text { building }\end{array} \\
\end{array}$ & $\begin{array}{r}36 \\
(5) \\
\end{array}$ & $\begin{array}{r}1.13 \\
(0.59) \\
\end{array}$ & (2) & $\begin{array}{c}\text { N Rectangular } \\
\text { building }\end{array}$ & $\begin{array}{r}47 \\
(13) \\
\end{array}$ & $\begin{array}{r}2.48 \\
(1.65) \\
\end{array}$ \\
\hline & & & & & & & & (2) & $\begin{array}{r}164 \\
(57) \\
\end{array}$ & $\begin{array}{r}2.36 \\
(1.51) \\
\end{array}$ & (1) & S Tower & $\begin{array}{r}137 \\
(35) \\
\end{array}$ & $\begin{array}{r}1.94 \\
(1.32) \\
\end{array}$ & (2) & N Tower & $\begin{array}{r}133 \\
(57) \\
\end{array}$ & $\begin{array}{r}2.57 \\
(1.32) \\
\end{array}$ \\
\hline & brick & $\mathrm{A}$ & \multicolumn{2}{|c|}{ Sanctuaries } & & & & B & \multicolumn{2}{|c|}{ Kraham } & $\mathrm{A}$ & \multicolumn{3}{|l|}{ N Library } & & & & \\
\hline & & & & & \multicolumn{3}{|c|}{ Inner enclosure } & & & & & & & & & & & \\
\hline \multirow{2}{*}{ Pr. Damrei } & laterite & & & & (1)-(2) & $\begin{array}{r}173 \\
(45)\end{array}$ & $\begin{array}{r}1.97 \\
(0.88)\end{array}$ & & & & & & & & & & & \\
\hline & brick & $\mathrm{B}$ & \multicolumn{2}{|c|}{ Sanctuary } & B & \multicolumn{2}{|c|}{ E\&W Gopuras } & & & & $\mathrm{B}$ & \multicolumn{3}{|l|}{ S Library } & $\mathrm{D}$ & \multicolumn{2}{|l|}{ N Library } & \\
\hline Pr. Chamreh & brick & B & \multicolumn{2}{|c|}{ Sanctuary } & & & & & & & & & & & & & & \\
\hline \multirow{2}{*}{ Pr. Krachap } & laterite & & & & (2)-(1) & $\begin{array}{l}117 \\
(73)\end{array}$ & $\begin{array}{r}2.01 \\
(1.47)\end{array}$ & (3) & $\begin{array}{r}776 \\
(173)\end{array}$ & $\begin{array}{r}2.19 \\
(1.10)\end{array}$ & & & & & & & & \\
\hline & brick & $\mathrm{C}$ & \multicolumn{2}{|c|}{ Sanctuary } & & & & & & & $\mathrm{C}$ & \multicolumn{3}{|l|}{ Libraries } & & & & \\
\hline \multirow[t]{2}{*}{ Pr. Kraham } & laterite & & & & & & & & & & (2) & EGopura & $\begin{array}{r}237 \\
(271)\end{array}$ & $\begin{array}{r}2.57 \\
(1.48) \\
\end{array}$ & & & & \\
\hline & brick & $\mathrm{C}$ & \multicolumn{2}{|c|}{ Sanctuary } & & & & & & & & & & & & & & \\
\hline \multirow[t]{2}{*}{ Pr. Chen } & laterite & (2) & $\begin{array}{r}190 \\
(220)\end{array}$ & \begin{tabular}{|r|}
2.75 \\
$(1.87)$ \\
\end{tabular} & (2) & $\begin{array}{r}270 \\
(208)\end{array}$ & \begin{tabular}{r|}
3.48 \\
$(2.27)$
\end{tabular} & \multirow[t]{2}{*}{ (3),(5) } & $\begin{array}{r}820 \\
(219)\end{array}$ & $\begin{array}{r}2.02 \\
(0.86)\end{array}$ & & & & & & & & \\
\hline & brick & & & & $\mathrm{B}$ & $\mathrm{E} \& \mathrm{~W} \mathrm{C}$ & opuras & & & & $\mathrm{B}$ & S Library & & & $\mathrm{D}$ & N Library & & \\
\hline $\begin{array}{l}\text { Pr. Neang } \\
\text { Khmau }\end{array}$ & laterite & (2) & $\begin{array}{r}74 \\
(22)\end{array}$ & \begin{tabular}{|r|}
5.21 \\
$(2.64)$ \\
\end{tabular} & (3) & $\begin{array}{r}549 \\
(151) \\
\end{array}$ & $\begin{array}{r}2.55 \\
(1.38) \\
\end{array}$ & & & & & & & & & & & \\
\hline Pr. D & laterite & (Sanct & $\begin{array}{l}\text { tuary } m: \\
\text { wood?) }\end{array}$ & nade of & (3),(5) & $\begin{array}{r}782 \\
(283) \\
\end{array}$ & $\begin{array}{r}2.01 \\
(1.05) \\
\end{array}$ & & & & & & & & & & & \\
\hline Pr. G & laterite & $\begin{array}{r}\text { (Sanct } \\
\text { sa }\end{array}$ & $\begin{array}{l}\text { tuary } m_{i} \\
\text { andston }\end{array}$ & $\begin{array}{l}\text { nade of } \\
\text { ne) }\end{array}$ & & & & & & & (3)-(2) & W Gopura & $\begin{array}{r}370 \\
(163)\end{array}$ & $\begin{array}{r}3.29 \\
(1.95) \\
\end{array}$ & & & & \\
\hline $\begin{array}{l}\text { Pr. Trapean } \\
\text { Rosei }\end{array}$ & laterite & $\begin{array}{r}\text { (Sanct } \\
\text { sa }\end{array}$ & $\begin{array}{l}\text { tuary } m i \\
\text { andston }\end{array}$ & $\begin{array}{l}\text { nade of } \\
\text { ne) }\end{array}$ & & & & & & & (3) & E Gopura & $\begin{array}{r}616 \\
(305)\end{array}$ & \begin{tabular}{|r|}
2.32 \\
$(1.30)$ \\
\end{tabular} & & & & \\
\hline Pr. Khtum & laterite & (3) & $\begin{array}{r}475 \\
(255)\end{array}$ & \begin{tabular}{|r|}
2.21 \\
$(1.18)$ \\
\end{tabular} & & & & & & & & & & & & & & \\
\hline Pr. Bak & laterite & (3) & $\begin{array}{r}463 \\
(310)\end{array}$ & \begin{tabular}{|r|}
2.08 \\
$(1.32)$ \\
\end{tabular} & & & & & & & & & & & & & & \\
\hline Pr. Banteay Pir & laterite & (3) & $\begin{array}{r}535 \\
(282)\end{array}$ & \begin{tabular}{|r|}
2.84 \\
$(1.36)$ \\
\end{tabular} & (4) & $\begin{array}{r}98 \\
(39)\end{array}$ & $\begin{array}{r}1.94 \\
(0.95) \\
\end{array}$ & (4) & $\begin{array}{r}184 \\
(73)\end{array}$ & $\begin{array}{r}1.67 \\
(0.82)\end{array}$ & & & & & & & & \\
\hline & brick & & & & & & & & & & $\mathrm{D}$ & N\&S Librar & & & $\mathrm{D}$ & Small towers & & \\
\hline Pr. Chrap & laterite & (3) & $\begin{array}{r}496 \\
(399) \\
\end{array}$ & \begin{tabular}{|r|}
2.49 \\
$(1.49)$ \\
\end{tabular} & (4) & $\begin{array}{r}220 \\
(103) \\
\end{array}$ & $\begin{array}{r}1.39 \\
(1.23)\end{array}$ & (5) & $\begin{array}{r}646 \\
(474) \\
\end{array}$ & $\begin{array}{r}1.72 \\
(1.27)\end{array}$ & & & & & & & & \\
\hline & brick & & & & & & & & & & D & N Library & & & & & & \\
\hline Pr. Pram & laterite & & & & (3),(5) & $\begin{array}{r}809 \\
(277)\end{array}$ & $\begin{array}{r}2.02 \\
(0.92) \\
\end{array}$ & & & & (5) & N Library & $\begin{array}{r}708 \\
(221)\end{array}$ & \begin{tabular}{|r|}
1.82 \\
$(0.60)$
\end{tabular} & & & & \\
\hline & brick & $\mathrm{D}$ & Sanctu & tuaries & & & & & & & $\mathrm{D}$ & S Library & & & & & & \\
\hline
\end{tabular}

\begin{tabular}{|c|c|c|c|}
\hline & & $* * *$ & $* * * *$ \\
\hline \multirow{5}{*}{$\begin{array}{l}\text { Construction } \\
\text { stages on the } \\
\text { basis of the } \\
\text { characteristics } \\
\text { of laterite }\end{array}$} & (1) & Sr: low & MS: low \\
\hline & (2) & Sr: low & MS: high \\
\hline & (3) & Sr: high & MS: high \\
\hline & (4) & Sr: low & MS: low \\
\hline & (5) & Sr: high & MS: low \\
\hline
\end{tabular}

\begin{tabular}{|l|l|}
\hline $\begin{array}{l}\text { Construction stages } \\
\text { deduced from the } \\
\text { characteristics of bricks and } \\
\text { laterite }\end{array}$ & $\begin{array}{l}\text { Stage A } \rightarrow \text { Stages B \& (1) } \rightarrow \\
\text { Stages C\&(2) } \rightarrow \text { Stages D\&(3) } \\
\rightarrow \text { Stage (4) } \rightarrow \text { Stage (5) }\end{array}$ \\
\hline
\end{tabular}

${ }^{*}$ Strontium content in ppm, ${ }^{* *}$ MS: Magnetic susceptibility of laterite $\left(\times 10^{-3} \mathrm{SI}\right.$ unit), ${ }^{* * *}$ low: lower than $300 \mathrm{ppm}$, high: higher than $400 \mathrm{ppm}$, and ${ }^{* * * *}$ low: lower than $2 \times 10^{-3}$ SI units, high: higher than $2 \times 10^{-3}$ SI units

Figure 5. A schematic showing the construction sequence of the laterite (Uchida et al., 2014) and brick buildings comprising the Koh Ker monuments, deduced from chemical compositions and magnetic susceptibilities of their building materials. 


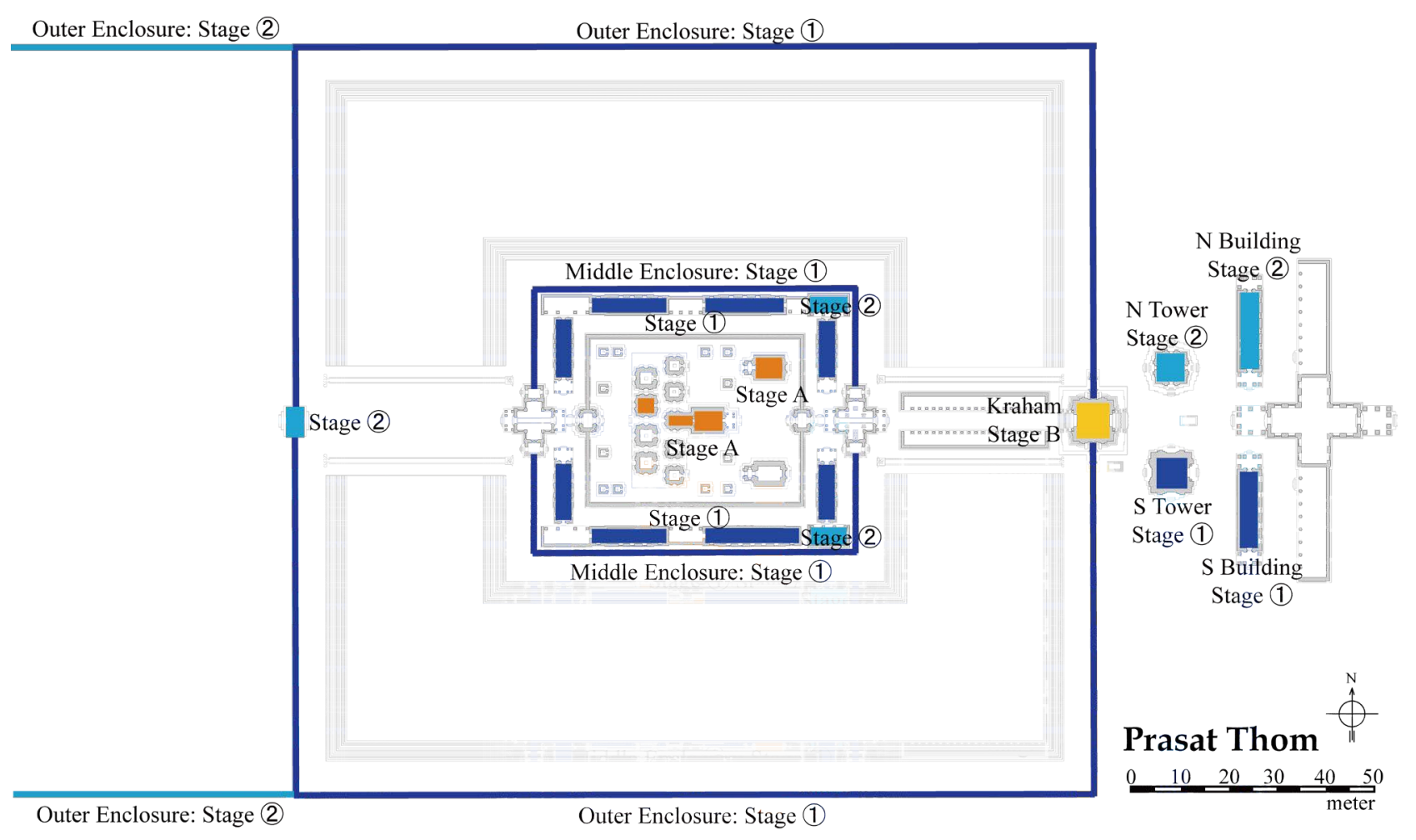

Figure 6. The construction sequence of the laterite and brick buildings in Prasat Thom of the Koh Ker monuments, deduced from the chemical compositions and magnetic susceptibilities of their building materials.

East Gopura (Prasat Kraham) (Stage B) of the Outer Enclosure was constructed almost at the same time as the laterite Outer Enclosure, belonging to Stage (1), giving an initial construction sequence of Stage A $\rightarrow$ Stage B \& Stage (1). The construction sequence of Stage B \& Stage (1) is also deduced from Pr. Damrei (Figure 7(f)).

The relationship between Stage C and Stage (2) is clearly evident in Prasat Krachap (Figure 7(a)). The brick Central Sanctuary is classified as Stage C, which is surrounded by the laterite Inner Enclosure, classified as Stage (2). This suggests that Stage C was almost contemporaneous with Stage (2). Although there is no temple that defines a relationship between Stage $\mathrm{C}$ and Stage (1), it is likely that Stage (1) preceded Stage C.

The relationship between Stage D and Stage (3) was deduced from buildings in Prasat Banteay Pir Chan (Figure 7(b)), Prasat Pram (Figure 7(c)), and Prasat Chrap (Figure 7(d)). In Prasat Banteay Pir Chan, the laterite Central Sanctuary (Stage (3) is surrounded by eight small brick towers, classified as Stage D. In Prasat Chrap, the three laterite Sanctuaries classified as Stage (3) and the Northern Library classified as Stage D, are all situated inside the Inner Enclosure (Stage (4)). This arrangement suggests Stage (3) of the laterite buildings preceded Stage D. However, in Prasat Pram, three brick Sanctuaries belonging to Stage D are surrounded by the Enclosure, classified as Stage (3). These relationships suggest that Stage (3) was contemporaneous with Stage D. 

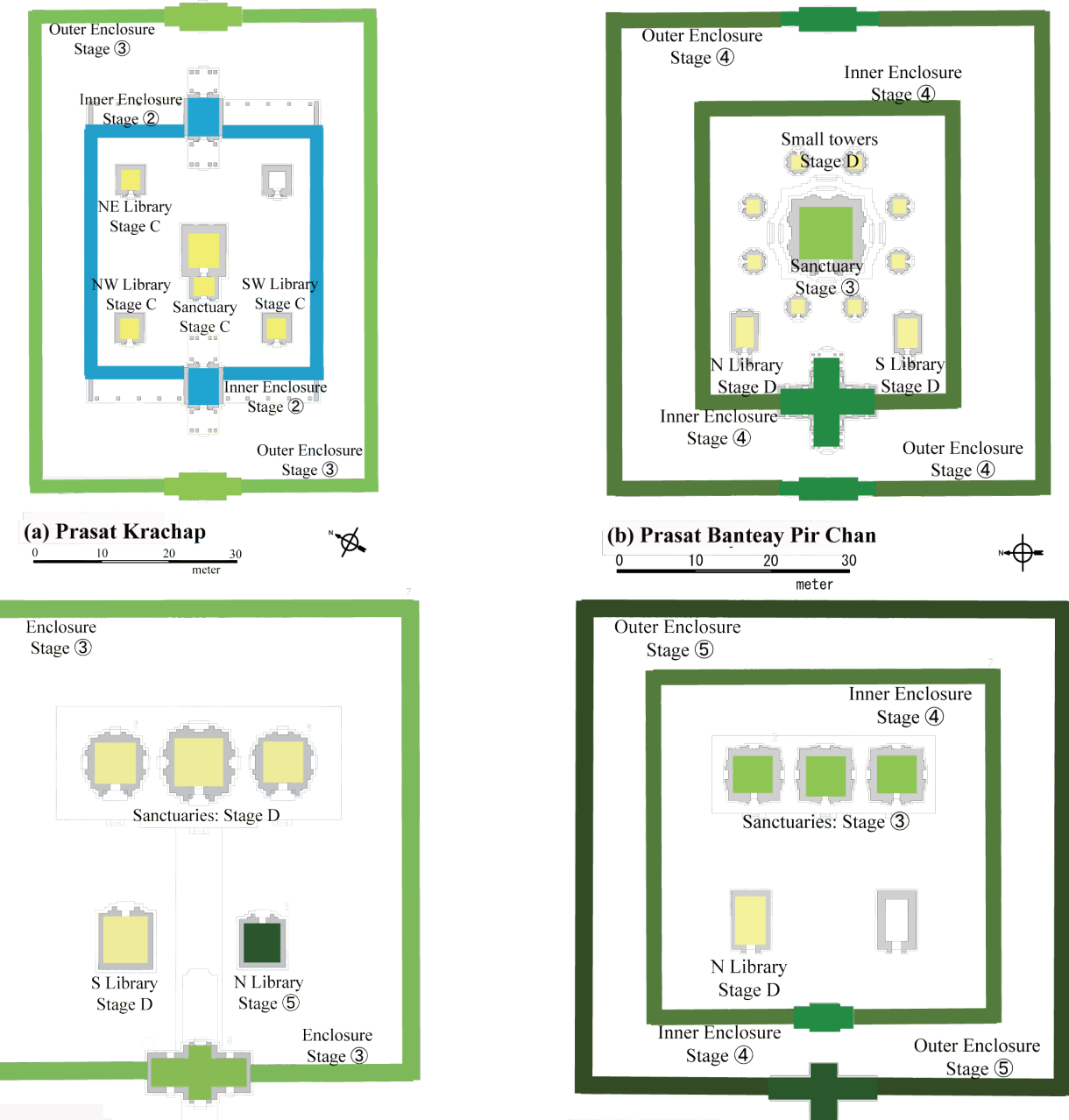

(c) Prasat Pram
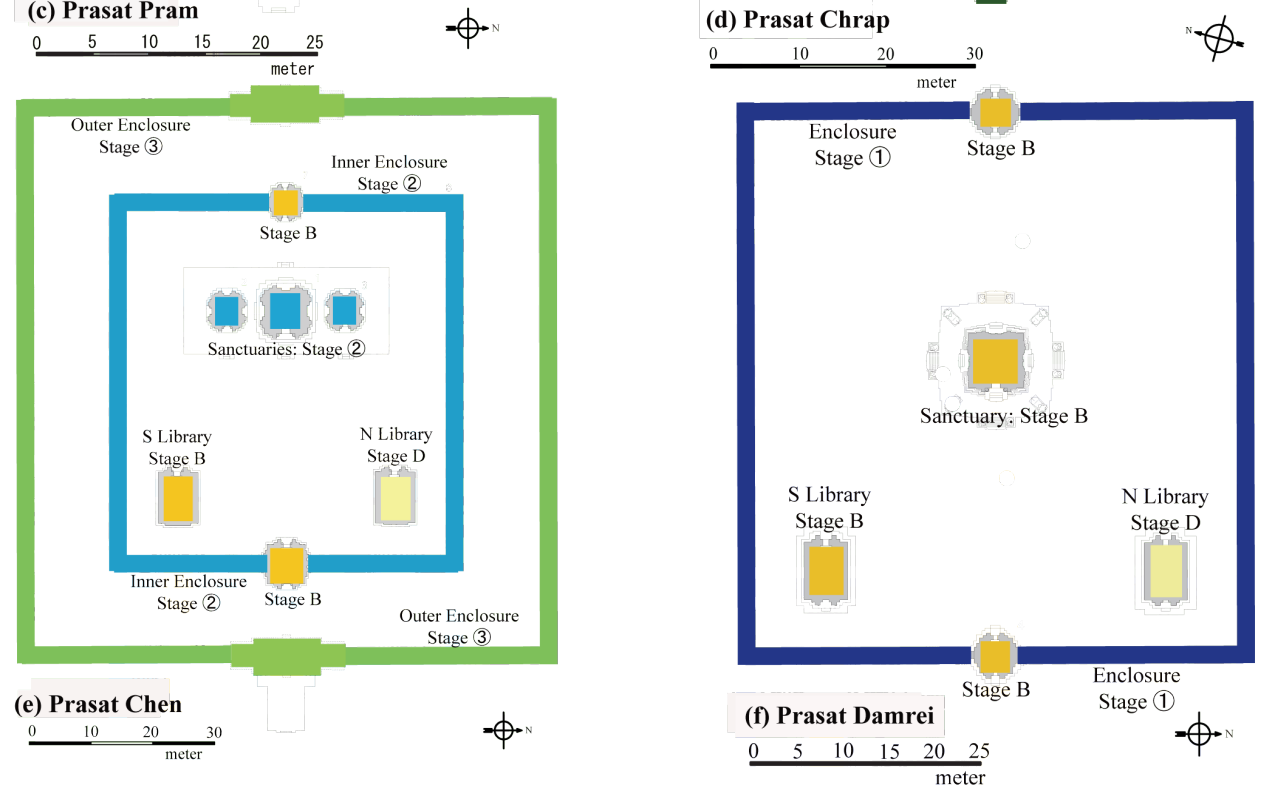

Figure 7. The construction sequence of the laterite and brick buildings in various major temples of the Koh Ker monuments, deduced from the chemical compositions and magnetic susceptibilities of their building materials. (a) Prasat Krachap; (b) Prasat Banteay Pir Chan; (c) Prasat Pram; (d) Prasat Chrap; (e) Prasat Chen; and (f) Prasat Damrei. 
From the above facts, the chronological relationship among Stages (1) to (5) for the laterite buildings and Stages $A$ to $D$ for the brick buildings is determined to be: Stage A $\rightarrow$ Stages B \& (1) $\rightarrow$ Stages C \& (2) $\rightarrow$ Stages D \& (3) $\rightarrow$ Stage (4) $\rightarrow$ Stage (5) (Figure 5). This result suggests that the Sanctuaries of Pr. Thom are the oldest ones in the Koh Ker monuments, followed by the Sanctuaries of Pr. Damrei and Pr. Chamreh. There is an apparent contradiction in timing of the construction of the Northern Libraries of Prasat Pram (Figure 7(c)), Prasat Chen (Figure 7(e)), and Prasat Damrei (Figure 7(f)). However, in other Khmer temples, the Northern Libraries were frequently added later (Uchida et al, 2003, 2007). Hence, the Northern Libraries are considered here to be later additions. Another contradiction arises in the construction of the Sanctuaries of Prasat Chen. The laterite Sanctuaries are classified as Stage (2), whereas the brick Southern Library, as well as East and West Gopuras of the Inner Enclosure are classified as Stage B. This contradicts the proposed construction sequence, relating Stages (1) to (5) and Stages A to D. To resolve this contradiction, we propose that the laterite Sanctuaries were constructed later to replace the old Sanctuaries (maybe originally built of wood or bricks). Alternatively, there is a possibility that a time gap between Stages B \& (1) and Stages C \& (2) is almost negligible.

\section{Conclusion}

Chemical analyses using a portable XRF analyzer and magnetic susceptibility measurements were conducted on bricks of nine temples in the Koh Ker monuments. Cluster analysis using magnetic susceptibility data and chemical compositional data for $\mathrm{Ti}, \mathrm{Fe}, \mathrm{Rb}, \mathrm{Y}$, and $\mathrm{Zr}$, grouped these brick buildings into four distinct groups, corresponding to Stages A to D. All buildings classified as Stage A are situated in Prasat Thom. Brick buildings of Prasat Damrei and Prasat Chen, and Prasat Kraham in Prasat Thom are classified as Stage B, while those of Prasat Chen and Prasat Kraham are classified as Stage C. Stage D includes the brick buildings of Prasat Banteay Pir Chan and Prasat Pram, and the Northern Libraries of Prasat Chen, Prasat Chrap and Prasat Damrei. Bricks used for each Stage have distinct chemical compositions: Stage A bricks are rich in $\mathrm{Y}$ (more than 33 ppm); Stage B bricks are rich in Rb (more than 39 ppm); Stage C bricks are rich in $\mathrm{Zr}$ (more than $540 \mathrm{ppm}$ ); Stage D bricks are depleted in Rb (less than $36 \mathrm{ppm})$.

Taking into consideration Stages (1) to (5) determined for the laterite buildings described in Uchida et al. (2014), a construction sequence for the brick buildings was determined based on chemical composition and magnetic susceptibility of their bricks, as well as the assumption that temples were constructed outwards from their center. We obtained a consistent construction sequence, except for the Central Sanctuary of Prasat Chen. The chronological relationship between Stages (1) to (5) for the laterite buildings and Stages A to D for the brick buildings of the Koh Ker monuments is defined as follows: Stage A $\rightarrow$ Stages B \& (1) $\rightarrow$ Stages C \& (2) $\rightarrow$ Stages D \& (3) $\rightarrow$ Stage (4) $\rightarrow$ Stage (5). The 
Northern Libraries of Prasat Pram, Prasat Chen and Prasat Damrei were likely added after the construction of the Inner Enclosure.

\section{Acknowledgements}

This research was carried out as part of the Japanese Government Team for Safeguarding Angkor, with permission from the Authority for the Protection and Management of Angkor and the Region of Siem Reap (APSARA Authority, Cambodia). It was supported financially by a Grant-in-Aid for Scientific Research from the Japan Society for the Promotion of Science (Project No. 23401001; E. Uchida).

\section{References}

Aymonier, É. (1900). Le Cambodge: I. Le royame actuel (pp. 397-411). Paris: Ernst Leroux.

Delaporte, L. (1880). Voyage au Cambodge. L'architecture khmère. Paris: Ch. Delagange, $462 \mathrm{p}$.

Dumarçay, J., \& Groslier, B. P. (1973). Le Bayon, histoire architecturale du temple, inscription du Bayon. Mémoire archéologiques, III-2, École Française d’Extrême-Orient, $332 \mathrm{p}$.

Evans, D. (2010-2011). The Archaeological Landscape of Koh Ker, Northwest Cambodia. Bulletin de l'École française d'Extrême-Orient, 97/98, 91-150. https://doi.org/10.3406/befeo.2010.6130

Groslier, G. (1924-1926). Promenades artistiques et archéologiques au Cambodge: 5. La region Nord-Est du Cambodge et son art. $A A K, 2,131-141$.

Harmand, J. (1879). Notes de voyage en Indo-Chine, Les Kouys. Ponthey Kakeh. Considération sur les monuments dits Khmer. Communication faite à la société académique indo-chinoise le 29 avril 1879. Annales de l'Extrême-Orient, 1, 329-337, 361-379.

Imai, N., Terashima, S., Itoh, S., \& Ando, A. (1995). 1994 Compilation Values for GSJ Reference Samples, “Igneous Rock Series”. Geochemical Journal, 29, 91-95. https://doi.org/10.2343/geochemj.29.91

Jacques, C., \& Lafond, P. (2004). L'Empire khmer. Cités et sanctuaries Ve-XIIIe siècles. Paris: Fayard, 275 p.

Lunet de Lajonquière, E. (1902). Inventaire descriptif des monuments du Cambodge. Paris: Publication de l'EFEO 4, E. Leroux, 355 p.

Mizoguchi, A., \& Nakagawa, T. (2011). Koh Ker and Beng Mealea. Two Large Monuments at the Eastern Portion of the Khmer Empire. Report for "Scientific Research Project on Provincial Ancient Khmer Cities and Temples", in 2007-2010, Grant-in-Aid for Scientific Research (A) by Japan Society for Promotion of Science, 238 p.

Parmentier, H. (1939). L'art Khmèr classique: Monuments du quadrant nord-est. PEFEO, 29, $187 \mathrm{p}$.

Uchida, E., \& Shiomoda, I. (2013). Quarries and Transportation Routes of Angkor Monuments Sandstone Blocks. Journal of Archaeological Science, 40, 1158-1164. https://doi.org/10.1016/j.jas.2012.09.036

Uchida, E., Cunin, O., Shimoda, I., Suda, C., \& Nakagawa, T. (2003). The Construction Process of the Angkor Monuments Elucidated by the Magnetic Susceptibility of Sandstone. Archaeometry, 45, 221-232. https://doi.org/10.1111/1475-4754.00105 
Uchida, E., Cunin, O., Suda, C., Ueno, A., \& Nakagawa, T. (2007). Consideration on the Construction Process and the Sandstone Quarries during the Angkor Period Based on the Magnetic Susceptibility. Journal of Archaeological Science, 34, 924-935.

https://doi.org/10.1016/j.jas.2006.09.015

Uchida, E., Tsuda, K., \& Shimoda, I. (2014). Construction Sequence of the Koh Ker Monuments in Cambodia Deduced from the Chemical Composition and Magnetic Susceptibility of Its Laterites. Heritage Science, 2, 1-10. 